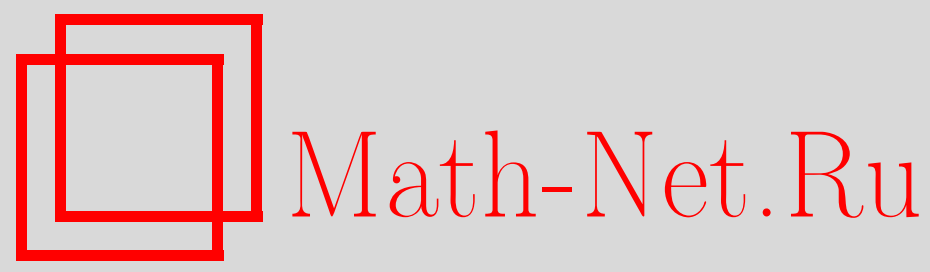

Х. Д. Икрамов, В. Н. Чугунов, Матричные задачи дополнения блочного типа, Матем. заметки, 2000, том 67, выпуск $6,863-873$

DOI: https://doi.org/10.4213/mzm905

Использование Общероссийского математического портала Math-Net.Ru подразумевает, что вы прочитали и согласны с пользовательским соглашением http://www . mathnet.ru/rus/agreement

Параметры загрузки:

IP: 34.229 .108 .108

26 апреля 2023 г., $13: 17: 43$

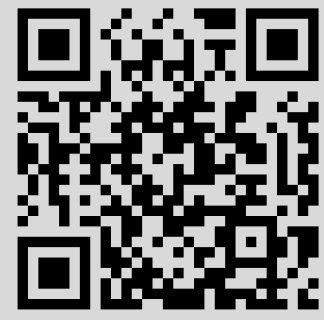




\title{
МАТРИЧНЫЕ ЗАДАЧИ ДОПОЛНЕНИЯ БЛОЧНОГО ТИПА
}

\author{
Х. Д. Икрамов, В.Н. Чугунов
}

Показана (безусловная или при определенных условиях) разрешимость трех матричных задач дополнения блочного типа. В задачах этого типа строится матрица $A$ с предписанным характеристическим многочленом, в которой заданы один или несколько блоков блочного $(2 \times 2)$-представления. В данном случае задаются соответственно: а) блок $A_{12} ;$ б) блок $A_{11}$; в) блоки $A_{11}$ и $A_{12}$. Обсуждается опыт реализации алгоритмов, решающих указанные задачи, в виде процедур системы символьных вычислений MAPLE.

Библиограффия: 6 названий.

1. Введение. Пусть фиксировано некоторое числовое поле $K$. Мы имеем в виду прежде всего поле $\mathbb{Q}$ рациональных чисел, но, в принципе, допустимо любое поле $x a$ рактеристики нуль.

Пусть выбран нормированный (т.е. имеющий старший коэффициент 1) многочлен степени $n$ с коэффициентами из поля $K$ :

$$
f(\lambda)=\lambda^{n}+a_{1} \lambda^{n-1}+\cdots+a_{n-1} \lambda+a_{n} .
$$

Пусть, далее, в блочном представлении $(n \times n)$-матрицы $A$ :

$$
A=\left(\begin{array}{ll}
A_{11} & A_{12} \\
A_{21} & A_{22}
\end{array}\right),
$$

заданы один или несколько блоков $A_{i j}$. Предполагается, что диагональные блоки $A_{11}$ и $A_{22}$ квадратные и элементы всех блоков суть числа из $K$. Требуется определить значения элементов незаданных блоков (иначе называемых свободным.ми) таким образом, чтобы характеристическим многочленом матрицы $A$ был многочлен (1.1). Этот класс обратных матричных задач на собственные значения называется задачами блочного muna.

Настоящая статья представляет собой продолжение нашей статьи [1], где был определен класс матричных задач дополнения. Задачи блочного типа могут рассматриваться как подмножество этого класса. Совокупность $\mathscr{K}$ пар индексов, характеризующая задачу дополнения, в случае блочных задач соответствует множеству элементов заданных блоков.

Работа первого автора выполнена при поддержке Российского фонда фундаментальных исследований, грант № 97-01-00927. 
Как и в [1], нас интересует вопрос о возможности решить конкретную задачу дополнения посредством конечной последовательности рациональных операций. Если задача разрешима в этом смысле, то алгоритм, реализующий ее решение в какой-либо системе безошибочных вычислений, будет давать точные результаты в случае рациональных или целочисленных входных данных.

Ниже мы рассматриваем три конкретные задачи дополнения блочного типа. В них задаются соответственно:

а) блок $A_{12}$;

б) блок $A_{11}$;

в) блоки $A_{11}$ и $A_{12}$.

Для краткости будем говорить об $A_{12}$-задаче, $A_{11}$-задаче и $\left(A_{11}, A_{12}\right)$-задаче.

Пусть $l$-порядок блока $A_{11}$ в (1.2). Тогда $m=n-l$ есть порядок блока $A_{22} . \mathrm{B}$ п. 2 . показано:

1) $A_{12}$-задача рационально разрешима для любого $l, 0<l<n$;

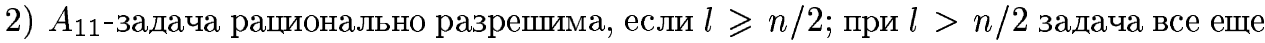
рационально разрешима, если в рациональной канонической форме (см. ниже) заданного блока $A_{11}$ число $k$ прямых слагаемых не превосходит $m$;

3) $\left(A_{11}, A_{12}\right)$-задача рационально разрешима, если пара $\left(A_{11}, A_{12}\right)$ вполне управляема. Последнее означает, что прямоугольная матрица

$$
R\left(A_{11}, A_{12}\right)=\left(\begin{array}{lllll}
A_{12} & A_{11} A_{12} & A_{11}^{2} A_{12} & \ldots & A_{11}^{l-1} A_{12}
\end{array}\right)
$$

имеет полньй строчный ранг $l$.

В п. 3 обсуждается наш опыт реализации алгоритмов, решающих указанные задачи, в виде процедур системы символьных вычислений MAPLE.

Закончим данный раздел напоминанием некоторых определений и фактов из теории матриц, важных для дальнейшего.

Сопоставим $(n \times n)$-матрице $A$ надполем $K$ характеристическую матрииу $\widehat{A}(\lambda)=$ $\lambda I_{n}-A$. Элементами последней являются (линейные) многочлены от $\lambda$, т.е. Элементы кольца многочленов $K[\lambda]$. Вьполняя в $K[\lambda]$ последовательность элементарных преобразований над строками и столбцами матрицы $\widehat{A}(\lambda)$, можно получить матрицу вида

$$
E(\lambda)=\left(\begin{array}{llll}
e_{1}(\lambda) & & & \\
& e_{2}(\lambda) & & \\
& & \ddots & \\
& & & e_{n}(\lambda)
\end{array}\right)
$$

где каждый из нормированных многочленов $e_{i}(\lambda)$ делит следующий многочлен $e_{i+1}(\lambda)$ :

$$
e_{i} \mid e_{i+1}, \quad i=1, \ldots, n-1 \text {. }
$$

Матрица (1.3) назьвается нормальной формой Смита, а многочлены $e_{1}(\lambda), \ldots, e_{n}(\lambda)-$ инвариантными множителями (или инвариантными многочленами) матрицы $A$.

Описание алгоритма построения формы Смита можно найти, например, в [2, ч. 1, разд. 3.22]. Из этого описания следует, в частности, что

$$
e_{i}(\lambda)=\frac{d_{i}(\lambda)}{d_{i-1}(\lambda)}, \quad i=1, \ldots, n,
$$


где $d_{0}(\lambda)=1$, a $d_{i}(\lambda)$ при $i \geqslant 1$ есть наибольший общий делитель миноров порядка $i$ матрицы $\widehat{A}(\lambda)$. Для нескалярной матрицы $A$, очевидно, $d_{1}(\lambda)=1$ и, в общем случае, ряд младших многочленов $d_{i}(\lambda)$ также равен 1 . Поэтому лишь некоторые из инвариантных многочленов $e_{i}(\lambda)$ не являются константами; число таких многочленов, из-за отсутствия общеупотребительного термина, будем называть жсрдановым индексом матрицы $A$. Многочлен $e_{n}(\lambda)$ - это минимальный многочлен матрищы $A$. Для простой матрицы минимальный многочлен совпадает с характеристическим, т.е. жорданов индекс такой матрицы равен 1 .

Если $(n \times n)$-матрицы $A$ и $B$ над полем $K$ имеют одну и ту же форму Смита $E(\lambda)$, то они подобны. Матрицу $T$, трансформирующую $B$ в $A=T B T^{-1}$, можно найти рациональным вычислением, если известны преобразования, переводящие характеристические матрицы $\widehat{A}(\lambda), \widehat{B}(\lambda)$ в $E(\lambda)$ (см. [2, ч. I, разд. 3.24]).

Матрица

$$
C(f)=\left(\begin{array}{cccc}
0 & 0 & \ldots & -a_{n} \\
1 & 0 & \ldots & -a_{n-1} \\
0 & 1 & \ldots & -a_{n-2} \\
\ldots & \ldots & \ldots & \ldots \\
0 & 0 & \ldots & -a_{1}
\end{array}\right)
$$

назьвается (правой) сопровождающей матрицей многочлена $f(\lambda)$. Другое название матрицы (1.5) - (правая) клетка Фробениуса.

Пусть $k$ - жорданов индекс матрицы $A$. Тогда $e_{1}(\lambda)=\cdots=e_{n-k}(\lambda)=1$. Положим

$$
f_{l}(\lambda)=e_{n-k+l}(\lambda), \quad l=1, \ldots, k
$$

Нетрудно проверить, используя формулу (1.4), что матрища

$$
F=C\left(f_{1}\right) \oplus C\left(f_{2}\right) \oplus \cdots \oplus C\left(f_{k}\right)
$$

имеет своей формой Смита матрицу $E(\lambda)$. Тем самым, матрицы $A$ и $F$ подобны. Матрица (1.6) назьвается в $[3$, гл. VI, $\S 6]$ первой естественной нормальной формой матрицы $A$. Однако мы будем вразрез с принятой (например, в [2]) и на наш взгляд ошшбочной терминологией называть $F$ рачиональной канонической формой для $A$, желая подчеркнуть, что $F$ можно получить из $A$ посредством рациональных вычислений.

2. Рациональная разрешимость. Пусть $K$ - произвольное поле характеристики нуль. В этом пункте показана (безусловная или при определенных условиях) рациональная разрешимость трех конкретных задач дополнения блочного типа. Каждой из них ниже отводится самостоятельный подпункт.

Сделаем несколько предварительных замечаний. Пусть $P$ и $Q$ - невырожденные матрицы порядка $l$ и $m$ соответственно. Если $A_{12}$-задача (рационально) разрешима для матрицы $A_{12}$, то она (рационально) разрешима и для матрицы $\widetilde{A}_{12}=P A Q$; разумеется, верно и обратное утверж дение. Действительно, если матрица (1.2) решает исходную задачу, то матрица

$$
\widetilde{A}=\left(P \oplus Q^{-1}\right) A\left(P^{-1} \oplus Q\right)=\left(\begin{array}{cc}
P A_{11} P^{-1} & P A_{12} Q \\
Q^{-1} A_{12} P^{-1} & Q^{-1} A_{22} Q
\end{array}\right)
$$


есть решение задачи с входным блоком $\widetilde{A}_{12}$. Это наблюдение позволяет при исследовании разрешимости $A_{12}$-задачи заменять заданную матрицу $A_{12}$ более простой (или в каком-то отношении более удобной) әквивалентной ей матрицей.

Точно так же в $A_{11}$-задаче заданную матрицу $A_{11}$ можно заменить на подобную матрицу $\widetilde{A}_{11}=P A_{11} P^{-1}$. Однако имеются и другие возможности.

Пусть матрища $(1.2)$ есть решение $A_{11}$-задачи. Для произвольной $(m \times l)$-матрищы $G$ положим

$$
F=\left(\begin{array}{cc}
I_{l} & 0 \\
G & I_{m}
\end{array}\right) \text {, }
$$

где $I_{l}$ обозначает единичную матрицу порядка $l$, и

$$
\begin{aligned}
\widetilde{A} & =F A F^{-1}=\left(\begin{array}{cc}
I_{l} & 0 \\
G & I_{m}
\end{array}\right) A\left(\begin{array}{cc}
I_{l} & 0 \\
-G & I_{m}
\end{array}\right) \\
& =\left(\begin{array}{cc}
A_{11}-A_{12} G & A_{12} \\
A_{21}-A_{22} G-G A_{12} G+G A_{11} & A_{22}+G A_{12}
\end{array}\right) .
\end{aligned}
$$

Таким образом, (рациональная) разрешимость исходной задачи переносится при любой матрице $G$ на задачу с входным блоком $A_{11}-A_{12} G$; при этом блок $A_{12}$ взят из решения (1.2) исходной задачи.

Сделанные замечания имеют силу и для $\left(A_{11}, A_{12}\right)$-задачи. В применении к ней первое замечание означает, что заданную пару $\left(A_{11}, A_{12}\right)$ можно заменить парой вида $\left(P A_{11} P^{-1}, P A_{12} Q\right)$. Согласно второму замечанию вместо $\left(A_{11}, A_{12}\right)$ может рассматриваться пара $\left(A_{11}-A_{12} G, A_{12}\right)$ с произвольной $(m \times l)$-матрицей $G . \quad \mathrm{B}$ отличие от $A_{11}$-задачи, блок $A_{12}$ известен с самого начала. Если задача решена для пары $\left(A_{11}-A_{12} G, A_{12}\right)$ и $\widetilde{A}-$ решение этой задачи, то, обращая преобразование $(2.1)$, придем к искомой матрище $A$.

2а. $A_{12}$-задача. Если $A_{12}=0$, то необходимьм и достаточньп условием разрешимости задачи является возможность разложить над $K$ заданньй многочлен $f(\lambda)$ в произведение

$$
f(\lambda)=f_{1}(\lambda) f_{2}(\lambda)
$$

многочленов степени $l$ и $m$ соответственно. Если разложение $(2.2)$ известно, то в качестве решения можно взять прямую сумму клеток Фробениуса: $A=C\left(f_{1}\right) \oplus C\left(f_{2}\right)$.

Если же $A_{12} \neq 0$, то $A_{12}$-задача всегда разрешима рационально. Действительно, пользуясь сделанным вьше замечанием, можно при необходимости заменить исходную задачу эквивалентной задачей, в которой входной $(l \times m)$-блок $\widetilde{A}_{12}$ имеет ненулевой элемент в позиции $(1, m)$. Окаймим $\widetilde{A}_{12}$ блоками

$$
\widetilde{A}_{11}=J_{l}^{T}, \quad \widetilde{A}_{22}=J_{m}^{T}, \quad \widetilde{A}_{22}=\left(\begin{array}{ccccc}
0 & 0 & \ldots & 0 & 1 \\
0 & 0 & \ldots & 0 & 0 \\
\ldots & \ldots & \ldots & \ldots \\
0 & 0 & \cdots & 0 & 0
\end{array}\right) .
$$

Символ $J_{s}-$ это стандартное обозначение (верхней) жордановой клетки порядка $s$ с нулевыми собственными значениями. 
Очевидно, что построенная матрица

$$
\widetilde{A}=\left(\begin{array}{ll}
\widetilde{A}_{11} & \widetilde{A}_{12} \\
\widetilde{A}_{21} & \widetilde{A}_{22}
\end{array}\right)
$$

имеет верхнюю форму Хессенберга. Ееведущая главная подматрица $\widetilde{A}_{n-1}$ порядка $n-1$ и последний столбец (взятьй без последней компоненты) составляют вполне управляемую пару

$$
\left(\begin{array}{ll}
\widetilde{A}_{n-1} & b_{n-1}
\end{array}\right) \text {. }
$$

Поэтому прямоугольную матрицу (2.4) можно приписыванием последней строки достроить до квадратной $(n \times n)$-матрицы $\widehat{A}$ так, чтобы характеристическим многочленом $\widehat{A}$ был заданный многочлен $f(\lambda)$. (Один из возможных методов достраивания был описан в $[1$, п. 2в].) Иначе говоря, изменяя элементы последней строки в матрице $(2.3)$, можно получить решение $A_{12}$-задачи.

В изложенной конструкции блок $\widetilde{A}_{12}$ был подчинен единственному требованию: неравенству нулю элемента $(1, m)$. Выбор той или иной формы для $\widetilde{A}_{12}$ с соблюдением данного требования может оказать значительное влияние на трудоемкость всего процесса. Мы вернемся к этому вопросу в п. 3.

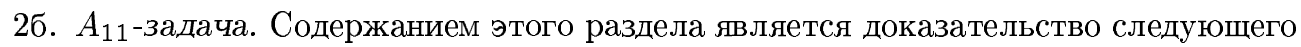
утверждения.

ТЕОРемА 1. Пусть $f(\lambda)$ - произвольный нормированный многочлен степени $n$ с коэффициентами из поля $K$. Пусть $l$ u $k$-порядок и жорданов индекс блока $A_{11}$ соответственно; $m=n-l$, причем $m>0$. Если $l \leqslant m$ или $l>m$, но $k \leqslant m$, то

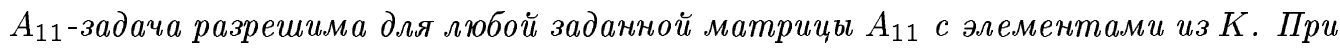
этом решение может быть построено конечными рачиональными вычислениями в поле $K$.

ДокАЗАТЕЛЬСТво теоремы будет получено адаптацией рассуждений в [4]. Заметим прежде всего, что при выдвинутых условиях на $l$ и $k$ существует $(l \times m)$-матрища $A_{12}$ такая, что $A_{11}$ и $A_{12}$ составляют вполне управляемую пару. Метод (рационального) построения $A_{12}$ будет описан в п. 3 .

Фиксируя одну из подходящих матриц $A_{12}$, будем с этого момента искать решение $\left(A_{11}, A_{12}\right)$-задачи. Замечание, сделанное в начале п. 2 , позволяет заменить $\left(A_{11}, A_{12}\right)$ парой $\left(A_{11}-A_{12} G, A_{12}\right)$, где $G$ - произвольная $(m \times l)$-матрица.

Пусть $x$ - произвольная линейная комбинация столбцов блока $A_{12}$ :

$$
x=A_{12} r, \quad r \in K^{m} \text {. }
$$

Известно, что для вполне управляемой пары $\left(A_{11}, A_{12}\right)$ матрицу $G$ можно выбрать таким образом, чтобы матрично-векторная пара $\left(A_{11}-A_{12} G, x\right)$ была вполне управляемой. Это утверждение в [4] обосновьвается ссылкой на [5]. Однако в п. 3 мы приведем явные формулы для элементов подходящей матрищы $G$.

Пусть матрица $G$ построена указанным образом по выбранному вектору $x$. Полагая $\widetilde{A}_{11}=A_{11}-A_{12} G$, перейдем от пары $\left(\widetilde{A}_{11}, A_{12}\right)$ к паре $\left(\widetilde{A}_{11}, A_{12} R\right)$, где $R$ - произвольная невырожденная $(m \times m)$-матрица с первым столбцом $r$. В новом блоке $\widetilde{A}_{12}=A_{12} R$ первым столбцом является вектор $x$. 
Приведем вполне управляемую пару $\left(\widetilde{A}_{11}, x\right)$ к канонической форме

$$
\left(F, e_{l}\right)
$$

посредством преобразования

$$
F=P \widetilde{A}_{11} P^{-1}, \quad e_{l}=P x .
$$

В формулах $(2.6),(2.7) e_{l}$ - это последний координатньй вектор пространства $K^{l}$, а $F-$ нижняя клетка Фробениуса:

$$
C(f)=\left(\begin{array}{ccccc}
0 & 1 & 0 & \ldots & 0 \\
0 & 0 & 1 & \ldots & 0 \\
\ldots \ldots & \ldots & \ldots & \ldots & \ldots \\
0 & 0 & 0 & \ldots & 1 \\
-a_{l} & -a_{l-1} & -a_{l-2} & \ldots & -a_{1}
\end{array}\right)
$$

Способ вычисления матрицы $P$, представляющий собой модификацию метода Данилевского, описан в $[1$, п. 3$]$.

Используем матрицу $P$ для еще одного преобразования задачи: от пары $\left(\widetilde{A}_{11}, \widetilde{A}_{12}\right)$ перейдем к паре $\left(P \widetilde{A}_{11} P^{-1}, P \widetilde{A}_{12}\right)=\left(F, \widehat{A}_{12}\right)$. Первьм столбцом блока $\widetilde{A}_{12}$, очевидно, является вектор $e_{l}$. Новьм переходом $\left(F, \widehat{A}_{12}\right) \longrightarrow\left(F-\widehat{A}_{12} G_{0}, \widehat{A}_{12}\right)$ аннулируем в блоке $(1,1)$ элементы нижней строки. Для этого достаточно взять матрицу $G_{0}$ вида

$$
G_{0}=\left(\begin{array}{ccc}
\dot{g}_{11} & \ldots & \dot{g}_{1 l} \\
0 & \ldots & 0 \\
\ldots & \ldots & \ldots \\
0 & \ldots & 0
\end{array}\right)
$$

с подходяшими элементами $\dot{g}_{11}, \ldots, \dot{g}_{1 l}$. Матрица $F-\widehat{A}_{12} G_{0}$ есть не что иное, как жорданова клетка $J_{l}$.

Подведем промежуточньй итог. Исходная $\left(A_{11}, A_{12}\right)$-задача разрешима, если разрешима задача для пары $\left(J_{l}, \widehat{A}_{12}\right)$.

Перейдем к решению последней. Недостающие блоки $\widehat{A}_{21}$ и $\widehat{A}_{22}$ для пары $\left(\widehat{A}_{11}, \widehat{A}_{12}\right)$ будем искать в виде

$$
\widehat{A}_{21}=\left(\begin{array}{cccc}
0 & 0 & \ldots & 0 \\
\ldots & \ldots & \ldots & \ldots \\
0 & 0 & \ldots & 0 \\
y_{1} & y_{2} & \ldots & y_{l}
\end{array}\right), \quad \widehat{A}_{22}=\left(\begin{array}{cccc}
0 & 1 & \ldots & 0 \\
\ldots & \ldots & \ldots & \ldots \\
0 & 0 & \ldots & 1 \\
z_{1} & z_{2} & \ldots & z_{m}
\end{array}\right)
$$

с неопределенными пока коэффициентами $y_{1}, y_{2}, \ldots, y_{l}$ и $z_{1}, z_{2}, \ldots, z_{m}$. В матрице

$$
\widehat{A}=\left(\begin{array}{cc}
J_{l} & \widehat{A}_{12} \\
\widehat{A}_{21} & \widehat{A}_{22}
\end{array}\right)
$$

ведущая главная подматрица порядка $n-1$ и последний столбец (без последней компоненты $z_{m}$ ) составляют вполне управляемую пару. Поэтому завершить построение $\widehat{A}$ (т.е. выбрать значения для $\left.y_{1}, y_{2}, \ldots, y_{l}, z_{1}, z_{2}, \ldots, z_{m}\right)$ можно так же, как в п. 2а. Теорема 1 доказана.

Мы стремились подчеркнуть конструктивность каждого этапа в предложенном доказательстве. Однако в рамках этой схемы эффективность процесса в целом можно значительно повысить, о чем будет сказано в п. 3.

2в. $\left(A_{11}, A_{12}\right)$-задача. По-прежнему все рассмотрения проводятся над полем $K$. 
Tеорема 2. Пусть $(l \times l)$-матрииа $A_{11} u(l \times m)$-матрииа $A_{12}$ составляют вполне управляемую пару. Тогда $\left(A_{11}, A_{12}\right)$-задача рачионально разрешима для любого нормированного многочлена $f(\lambda)$ степени $n, n=l+m$.

Это утверждение было установлено в ходе доказательства теоремы 1.

3. Программная реализация. Для каждой из задач $2 \mathrm{a}-2$ в нами составлена процедура на языке системы символьных вычислений MAPLE. Будем назьвать алгоритмы, реализованные этими процедурами, алгоритмами 1-3.

Алгоритмы 1-3, сохраняя схемы доказательств из п. 2, повьшают численную эффективность отдельных этапов и сушественно снижают трудоемкость процесса в целом.

Пусть $r$ - ранг заданного блока $A_{12}$ в задаче 2 a. Исходная задача заменяется эквивалентной с блоком $\widetilde{A}_{12}=P A_{12} Q$ вида

$$
\widetilde{A}_{12}=\left(\begin{array}{cc}
0 & \mathscr{P}_{r} \\
0 & 0
\end{array}\right)
$$

Здесь $\mathscr{P}_{r}$ - это специальная матрица-перестановка:

$$
\mathscr{P}_{r}=\left(\begin{array}{llll} 
& & & 1 \\
& & & \\
& & & \\
1 & & &
\end{array}\right)
$$

Такой выбор блока $\widetilde{A}_{12}$ значительно упрошает приведение пары $(2.4)$ к канонической форме; это приведение (описываемое в [1, п. 2в]) является главным этапом в достраивании пары до матрицы с требуемьм характеристическим многочленом.

Результаты численных экспериментов с алгоритмом 1 представлены в табл. 1. Для вычислений использовался персональный компютер класса Intel Pentium Pro с тактовой частотой 166 Мг и оперативной памятью 32 Мб. Входными данньми задач (т.е. элементами блока $A_{12}$ и коэффициентами $a_{1}, a_{2}, \ldots, a_{n}$ предписьваемого характеристического многочлена) служили целые числа, (псевдо) случайным образом выбираемые из отрезка $[-9,9]$. Значения порядка $n$, указываемые в первом столбце таблицы, менялись от 4 до 40 с шагом 4. Поскольку трудоемкость алгоритма зависит не только от $n$, но и от размера $l \times m$ блока $A_{12}$, то при каждом значении $n$ решались задачи для значений $l=1, n / 4, n / 2,3 n / 4, n-1$. Во втором-шестом столбцах таблицы приведено время (в секундах), полученное для различных пар $(n, l)$.

Число $S_{1}$ арифметических операций в алгоритме 1 выражается многочленом третьей степени от $n, l$ и $r$; старшие члены этого многочлена имеют вид

$$
5 n^{3}+\left(n+l m+m^{2}\right) r-(n+m) \frac{r^{2}}{2}
$$

(напомним, что $m=n-l$ ). Для псевдослучайной матрицы $A_{12}$, как правило, имеем $r=\min \{l, m\}$. Принимая это равенство и фиксируя $n$, можем показать, что график 


\begin{tabular}{|c||c|c|c|c|c|}
\hline \multicolumn{1}{|c||}{} & \multicolumn{5}{c|}{$l$} \\
\cline { 2 - 6 }$n$ & 1 & $n / 4$ & $n / 2$ & $3 n / 4$ & $n-1$ \\
\hline 4 & 0.8 & 0.9 & 0.9 & 0.9 & 0.8 \\
8 & 1.7 & 1.8 & 2.1 & 1.9 & 1.8 \\
12 & 3.4 & 4.0 & 4.9 & 4.1 & 3.4 \\
16 & 6.3 & 7.9 & 10.1 & 8.1 & 6.1 \\
20 & 10.6 & 14.4 & 18.5 & 14.6 & 10.2 \\
24 & 18.3 & 23.6 & 31.2 & 23.8 & 15.8 \\
28 & 25.0 & 37.4 & 50.1 & 38.1 & 23.5 \\
32 & 35.4 & 55.4 & 76.5 & 57.6 & 33.1 \\
36 & 48.8 & 79.1 & 110.5 & 80.9 & 45.5 \\
40 & 65.2 & 110.1 & 157.8 & 110.9 & 60.7 \\
\hline
\end{tabular}

ТАБЛ. 1

функции $S_{1}=S_{1}(l)$ приблизительно симметричен относительно прямой $l=n / 2$; кроме того, прямая $l=n / 2$ проходит вблизи точки глобального максимума $S_{1}$. Обе эти закономерности очень точно отражены строками табл. 1.

Первым этапом алгоритма 2 является построение $(l \times m)$-матрицы $A_{12}$, составляюшей вместе с заданной матрищей $A_{11}$ вполне управляемую пару. Это делается следующим образом. Матрица $A_{11}$ приводится к (транспонированной) рациональной канонической форме:

$$
A_{11} \longrightarrow \breve{A}_{11}=P A_{11} P^{-1}=C^{T}\left(f_{1}\right) \oplus C^{T}\left(f_{2}\right) \oplus \cdots \oplus C^{T}\left(f_{k}\right)
$$

(Почему вместо правых клеток Фробениуса $C\left(f_{i}\right)$ нам нужны нижние клетки, будет ясно из дальнейшего.) Приведение (3.1) можно осуществить с помощью библиотечной функции frobenius системы MAPLE. Эта функция входит в состав пакета по линейной алгебре linalg и вычисляет обычную (т.е. правую) каноническую форму. Чтобы получить (3.1), нужно применить frobenius к транспонированной матрице $A_{11}^{T}$.

Обозначим через $s_{i}(1 \leqslant i \leqslant k)$ порядок матрицы

$$
C^{T}\left(f_{1}\right) \oplus C^{T}\left(f_{2}\right) \oplus \cdots \oplus C^{T}\left(f_{i}\right)
$$

Символ $e_{j}$ ниже обозначает $j$-й координатньй вектор размерности $l$. Легко видеть, что для матрицы

$$
\breve{A}_{12}=\left(\begin{array}{lllllll}
e_{s_{1}} & e_{s_{2}} & \ldots & e_{l} & 0 & \ldots & 0
\end{array}\right)
$$

где число столбцов равно $m$, пара $\left(\breve{A}_{11}, \breve{A}_{12}\right)$ является вполне управляемой. Напомним, что по предположению $m \geqslant k$.

На втором этапе алгоритма полагаем $x=e_{l}$. Тогда $(m \times l)$-матрищу $G=\left(g_{i j}\right)$ можно определить так: для $t=1,2, \ldots, k$

$$
g_{t j}= \begin{cases}\left\{\widetilde{A}_{11}\right\}_{s_{t}, j}, & s_{t-1}+1 \leqslant j \leqslant s_{t} \\ -1, & j=s_{t}+1\end{cases}
$$


Все прочие элементы матрицы $G$ равны нулю. В первой из формул (3.2) при $t=1$ следует считать $s_{0}=0$; вторая формула (3.2) не применяется при $t=k$. В качестве примера предположим, что $l=5, m=3, k=2$ и матрища $\breve{A}_{11}$ имеет вид

$$
\left(\begin{array}{ccccc}
0 & 1 & 0 & 0 & 0 \\
-a_{2} & -a_{1} & 0 & 0 & 0 \\
0 & 0 & 0 & 1 & 0 \\
0 & 0 & 0 & 0 & 1 \\
0 & 0 & -b_{3} & -b_{2} & -b_{1}
\end{array}\right)
$$

В таком случае

$$
G=\left(\begin{array}{ccccc}
-a_{2} & -a_{1} & -1 & 0 & 0 \\
0 & 0 & -b_{3} & -b_{2} & -b_{1} \\
0 & 0 & 0 & 0 & 0
\end{array}\right)
$$

Нетрудно проверить, что матрища $\breve{A}_{11}-\breve{A}_{12} G$ есть жорданова клетка $J_{5}$. Точно так жеи в общем случае указанный выбор матрицы $G$ обеспечивает равенство $\breve{A}_{11}-\breve{A}_{12} G=J_{l}$. Пара $\left(J_{l}, \breve{A}_{12}\right)$ соответствует по своей форме паре $\left(J_{l}, \widehat{A}_{12}\right)$ в доказательстве теоремы 1. Это означает, что преобразование (2.7) из п. $2 б$ и два следующих за ним вьполнять не нужно.

\begin{tabular}{|c||c|c|c|c|c|}
\hline \multicolumn{1}{|c||}{} & \multicolumn{5}{c|}{$l$} \\
\cline { 2 - 6 }$n$ & 1 & $n / 4$ & $n / 2$ & $3 n / 4$ & $n-1$ \\
\hline 4 & 1.5 & 1.6 & 1.6 & 1.6 & 1.6 \\
8 & 2.7 & 2.8 & 2.9 & 3.7 & 3.4 \\
12 & 4.9 & 5.2 & 5.6 & 6.7 & 8.1 \\
16 & 8.5 & 9.0 & 9.8 & 13.3 & 20.9 \\
20 & 13.6 & 14.5 & 16.6 & 27.6 & 66.3 \\
24 & 20.0 & 21.8 & 25.1 & 57.7 & 188.8 \\
28 & 29.2 & 30.8 & 40.1 & 130.1 & 513.4 \\
32 & 40.3 & 43.4 & 63.2 & 262.1 & 1256.7 \\
36 & 54.2 & 57.8 & 93.8 & 546.1 & 2878.0 \\
40 & 72.4 & 81.6 & 143.5 & 1069.7 & 6170.4 \\
\hline
\end{tabular}

ТАБл. 2

Табл. 2 содержит результаты наших экспериментов с алгоритмом 2. Она построена аналогично табл. 1. Можно показать, что число $S_{2}$ арифметических операций в алгоритме 2 является многочленом третьей степени от $n$ и $l$, причем два старших члена этого многочлена имеют вид

$$
9 n^{3}+n l^{2}
$$

Таким образом, при фиксированном $n$ функция $S_{2}=S_{2}(l)$ монотонно возрастает. Строки табл. 2 отражают эту монотонность поведения. Вместе с тем при увеличении $l$ от 1 до $n-1$ коэффициент роста $S_{2}$ согласно (3.3) не должен превышать $10 / 9$. Совсем иной характер возрастания $S_{2}$ демонстрирует табл. 2 . Это объясняется следующим образом: 
формула (3.3) получена в предположении, что алгоритм приведения матрицы к рациональной канонической форме реализован "грамотно"; в таком случае его трудоемкость выражается величиной $O\left(l^{3}\right)$ (напомним, что в задаче $2 б$ к рациональной форме приводится $(l \times l)$-матрица $\left.A_{11}\right) . \mathrm{K}$ сожалению, процедура frobenius реализует указанньй алгоритм весьма неэффективно. Мы установили экспериментально, что время обработки $(l \times l)$-матрищы этой процедурой ведет себя как $O\left(l^{4}\right)$. С поправкой на данное обстоятельство и нужно воспринимать результаты в табл. 2 .

В алгоритме 2 удалось существенно сократить объем вычислений за счет специального выбора блока $A_{12}$. Этого нельзя сделать в алгоритме 3 , поскольку здесь блок $A_{12}$ задан условиями задачи. В целом алгоритм 3 можно рассматривать как (почти) точную реализацию схемы, использованной в доказательстве теоремы 1 . Введен только один дополнительньй этап, на котором исходная пара $\left(A_{11}, A_{12}\right)$ преобразуется к виду

$$
\begin{aligned}
& \breve{A}_{11}=Q A_{11} Q^{-1}=\left(\begin{array}{cccc}
F_{11} & F_{12} & \ldots & F_{1 u} \\
& F_{22} & \ldots & F_{2 u} \\
\ldots & \ldots & \ldots \\
& & F_{u u}
\end{array}\right) \text {, } \\
& \breve{A}_{12}=Q A_{12}=\left(\begin{array}{ccccccc}
f_{11} & f_{12} & \ldots & f_{1 u} & f_{1, u+1} & \ldots & f_{1 m} \\
& f_{22} & \ldots & f_{2 u} & f_{2, u+1} & \ldots & f_{2 m} \\
& & \ldots & \ldots & \ldots & \ldots & \ldots \\
& & & f_{u u} & f_{u, u+1} & \ldots & f_{u m}
\end{array}\right) \text {. }
\end{aligned}
$$

В этих формулах $F_{11}, F_{22}, \ldots, F_{u u}$ суть правые клетки $Ф$ робениуса, порядки которых обозначим через $\alpha_{1}, \alpha_{2}, \ldots, \alpha_{u}\left(\alpha_{1}+\alpha_{2}+\cdots+\alpha_{u}=l\right)$; тогда $f_{11}, f_{22}, \ldots, f_{u и}$-это первые координатные векторы размерности $\alpha_{1}, \alpha_{2}, \ldots, \alpha_{u}$ соответственно. Возможность приведения вполне управляемой пары $\left(A_{11}, A_{12}\right)$ к форме $(3.4),(3.5)$ показана, например, в [6, теорема 1.6]. Средством ее выгисления может быть алгоритм Данилевского. Для пары $\left(\breve{A}_{11}, \breve{A}_{12}\right)$ в форме $(3.4),(3.5)$ можно предложить простую матрицу $G$, отвечающую выбору в качестве вектора $(2.5)$ первого столбца блока $\breve{A}_{12}$, т.е. координатного $l$-мерного вектора $e_{1}$. При таком векторе $x$ не понадобится правостороннее преобразование с матрицей $R$ (см. п. 2б).

Для $t=1,2, \ldots, k-1$ положим $s_{t}=\alpha_{1}+\alpha_{2}+\cdots+\alpha_{t}$ и $g_{t+1, s_{t}}=-1$. Все остальные элементы матрицы $G$ равны нулю. Легко проверить, что такому выбору $G$ соответствует верхняя хессенбергова матрища $\widetilde{A}_{11}=\breve{A}_{11}-\breve{A}_{12} G$. Очевидно, что матрично-векторная пара $\left(\widetilde{A}_{11}, e_{1}\right)$ вполне управляема.

Результаты численных экспериментов с алгоритмом 3 показаны в табл. 3 . Она устроена так же, как и две предыдущих.

При фиксированном $n$ число $S_{3}=S_{3}(l)$ арифметических операций в алгоритме 3 выражается формулой

$$
S_{3}(l)= \begin{cases}\frac{7}{3} l^{3}+n l^{2}+10 \frac{1}{3} n^{3}+O\left(n^{2}\right), & l<n-1, \\ 12 \frac{1}{3} n^{3}+O\left(n^{2}\right), & l=n-1 .\end{cases}
$$

Для $l<n-1$ функция $S_{3}(l)$ монотонно возрастает вместе с $l$. Это поведение отражено строками табл. 3. Однако, при $l=n-1$ происходит “скачок вниз". Он связан с тем, что пара (3.4), (3.5) в этом случае представляет собой клетку Фробениуса порядка $n-1$ 


\begin{tabular}{|c||c|c|c|c|c|}
\hline \multicolumn{1}{|c||}{} & \multicolumn{5}{c|}{$l$} \\
\cline { 2 - 6 }$n$ & 1 & $n / 4$ & $n / 2$ & $3 n / 4$ & $n-1$ \\
\hline 4 & 1.6 & 1.5 & 1.6 & 1.6 & 1.6 \\
8 & 2.8 & 3.0 & 3.6 & 4.8 & 4.1 \\
12 & 5.4 & 6.0 & 8.4 & 25.9 & 13.4 \\
16 & 9.0 & 11.3 & 25.8 & 168.2 & 56.3 \\
20 & 14.5 & 20.1 & 91.9 & 923.5 & 224.1 \\
24 & 21.4 & 34.7 & 347.9 & 3995.0 & 690.7 \\
28 & 31.6 & 58.0 & 1075.3 & & 2076.5 \\
32 & 44.1 & 102.6 & 3320.1 & & \\
36 & 59.7 & 198.3 & 8480.8 & & \\
40 & 77.3 & 373.1 & & & \\
\hline
\end{tabular}

ТАБл. 3

и единичный вектор $e_{1}$ размерности $n-1$. Построение матрищы $G$ и соответствующее преобразование здесь не нужны, что и объясняет неравенство

$$
S_{3}(n-1)<\lim _{l \rightarrow(n-1)-0} S_{3}(l)
$$

\section{СПИСОК ЦИТИРОВАННОЙ ЛИТЕРАТУРЫ}

[1] Икрамов Х. Д., Чугунов В.Н. Матричные задачи дополнения с произвольным расположением заданных элементов // ЖВМиМФ. 1999. Т. 39. № 9. С. 1427-1444.

[2] Маркус М., Минк Х. Обзор по теории матриц и матричных неравенств. М.: Наука, 1972.

[3] Гантмахер Ф.Р. Теория матриц. М.: Наука, 1966.

[4] Wimmer H. K. Existenzsätze in der Theorie der Matrizen und lineare Kontrolltheorie // Monatsh. Math. 1974. V. 78. P. 256-263.

[5] Wonham W. M., Morse A. S. Decoupling and pole assignment in linear multivariable systems: a geometric approach // SIAM J. Control. 1970. V. 8. P. 1-18.

[6] Икрамов Х. Д. О размещении полюсов линейных стационарных систем // Вычисл. процессы и системы. Вып. 9. М.: Наука, 1993. С. 35-162.

(Х. Д. Икрамов) Московский государственный университет им. М. В. Ломоносова Поступило

(В.Н. Чугунов) Институт выгислительной математики РАН 05.04.1999 